\title{
Measurement of ocular torsion variation following superior oblique tenectomy
}

\section{Medida da variação da torção ocular após a tenectomia do oblíquo superior}

\author{
Bruna lana Ducca ${ }^{1,2}$, Carlos Ramos de Souza-Dias ${ }^{3}$, Aline Cristina Fioravantı Lui ${ }^{4}$, Mauro Goldchmit ${ }^{1,2}$
}

\begin{abstract}
Purposes: To objectively evaluate the torsional effect of the superior oblique muscle-weakening surgery using the tenectomy technique proposed by Souza-Dias. Methods: The present prospective study included 10 patients (20 eyes) with horizontal strabismus, bilateral superior oblique overaction and A-pattern of 15 to 30 prism diopters who underwent superior oblique tenectomy. Objective assessment of ocular torsion was performed by retinography immediately before and one month after surgery. The amount of ocular torsion was determined by measuring the angle formed by a horizontal line drawn across the geometric center of the optic disc and a second line connecting this point to the fovea.

Results: The median preoperative angle was $5.56^{\circ}$ in the right eyes and $-3.43^{\circ}$ in the left eyes. The median postoperative angle was $1.84^{\circ}$ in the right eyes and $-3.12^{\circ}$ in the left eyes. The angle variation was statistically significant in both eyes ( $p=0.012$ and $p=0.01$, respectively).

Conclusion: The present study suggests that superior oblique tenectomy has an extorter effect, decreasing the intorsion detected on overaction of this muscle.

Keywords: Strabismus; Oculomotor muscles; Torsion abnormality; Ophthalmologic surgical procedures/methods
\end{abstract}

\section{RESUMO}

Objetivo: Avaliar, de forma objetiva, a torção ocular após a tenectomia do oblíquo superior, proposta por Souza-Dias.

Métodos: Estudo prospectivo de dez pacientes (20 olhos) com estrabismo horizontal, hiperfunção bilateral dos oblíquos anisotropia em A de 15 a 30 dioptrias prismáticas submetidos à tenectomia bilateral dos oblíquos superiores. A avaliação objetiva da torção foi realizada com a retinografia antes e após a cirurgia, determinando-se o ângulo de torção formado entre a linha horizontal que passa pelo centro do disco óptico e a linha que passa pelo centro da fóvea.

Resultados: A mediana dos ângulos de torção pré-operatória foi de $5,56^{\circ}$ nos olhos direitos e de $3,43^{\circ}$ nos esquerdos. Após a cirurgia, o ângulo mediano foi de 1,84 nos olhos direitos e de $-3,12^{\circ}$ nos esquerdos. Em ambos os olhos, a variação absoluta foi estatisticamente significativa ( $p=0,012 / p=0,01)$.

Conclusão: Opresente estudo demonstrou que a tenectomia do oblíquo superior tem ação extorsora, reduzindo de forma significante a intorção encontrada nos casos de hiperfunção deste músculo.

Descritores: Estrabismo/cirurgia; Músculos oculomotores; Anormalidade torcional; Procedimentos cirúrgicos oftalmológicos/métodos

\section{INTRODUCTION}

Eye torsion can be measured both subjectively and objectively. Subjective measurement is obtained using the Maddox rod test, which corresponds to the patient's perception of image torsion. Objective measurement is performed using funduscopy or fundus photography (retinography) $)^{(1-5)}$.

Superior oblique overaction is recognized by some authors as a major factor in the genesis of A-pattern and in the intorsion observed in the fundus ${ }^{(6,7)}$. Correction is obtained using superior oblique surgical weakening, which can be performed through the medial approach (lengthening, tenotomy ${ }^{(8)}$ or Berke tenectomy $\left.{ }^{(9)}\right)$, or by the lateral approach to the superior rectus muscle (posterior partial tenectomy ${ }^{(10)}$, disinsertion ${ }^{(7)}$, or tenectomy $\left.{ }^{(11)}\right)$. Procedures that weaken anterior fibers result in a greater excycloduction ${ }^{(6,7)}$.

The cycloduction that results from weakening surgeries of the inferior oblique muscle is widely reported in the literature ${ }^{(2,5,12,13)}$. However, few studies have evaluated the torsional effect resulting from weakening procedures involving the superior oblique muscle. Sharma et al. ${ }^{(14)}$ studied objective and subjective torsion variation in 15 patients with superior oblique overaction who underwent partial posterior tenectomy. Mean preoperative torsion was $6.46^{\circ} \pm 3.44^{\circ}$ in the right eyes and $7.13^{\circ} \pm 2.56^{\circ}$ in the left eyes. At the postoperative evaluation, the mean correction was found to be $0.06^{\circ} \pm 1.10^{\circ}$ in the right eyes and $0.46^{\circ} \pm 0.91^{\circ}$ in the left eyes. Roizen et al. ${ }^{(15)}$ performed a subjective evaluation (Maddox rod test) of five patients with intorsion and diplopia who underwent superior oblique anterior tenectomy; the mean correction of torsion was $5.2^{\circ}$.

No studies were found that included an objective evaluation of the cycloduction obtained with superior oblique full tenectomy. The aim of the present study was to evaluate eye torsion after weakening surgery of the superior oblique muscle using the tenectomy technique proposed by Souza-Dias ${ }^{(11)}$.

\section{METHODS}

The protocol of the present study was approved by the Committee on Ethical Research of the Irmandade da Santa Casa de Misericórdia Hospital of São Paulo (protocol \#044/10). Informed consent was obtained from patients or their responsible caretaker before surgery.

A prospective study was performed that included 10 patients with strabismus and bilateral superior oblique overaction $(2+-3+$ in a scale of $1+-4+$ ) whose surgical planning included bilateral superior

Submitted for publication: February 6, 2014

Study conducted at Department of Ophthalmology, Santa Casa de Misericórdia de São Paulo, São Paulo, SP, Brazil.

Strabismus Section, Department of Ophthalmology, Santa Casa de Misericórdia de São Paulo, São Paulo, SP, Brazil.

Strabismus Section, Cema Hospital, São Paulo, SP, Brazil.

3 Ophthalmology Department, Santa Casa de Misericórdia de São Paulo, São Paulo, SP, Brazil.

Uveitis Section, Department of Ophthalmology, Santa Casa de Misericórdia de São Paulo, São

Paulo, SP, Brazil.

Funding: No specific financial support was used for this study.

Disclosure of potential conflicts of interest: None of the authors have any potential conflicts of interest to disclose.

Corresponding author: Bruna Lana Ducca. Rua Brás Cardoso, 85/101 - São Paulo, SP - 04510-030 Brazil - E-mail: brunaducca@yahoo.com 
oblique tenectomy; in this technique proposed by Souza-Dias ${ }^{(11)}$ $6 \mathrm{~mm}$ of the SO tendon was resected from its insertion. Patients with nystagmus, those who did not permit the performance of the retinography and/or those submitted to a previous eye surgery were excluded from the present study. The mean age of the study group was 21.3 years (range four to 28 years). Four patients were male (40\%) and six were female (60\%). Eight patients had esotropia (80\%) and two had exotropia (20\%).

All patients underwent ophthalmological examination that included visual acuity measurement (Snellen table), cycloplegic refractometry, biomicroscopy, and fundus examination. Measurement of the deviation was performed using the alternate cover test with prisms for distance in the primary, superior and inferior positions, and for near. All had A-pattern varying from 15 prism diopters (PD) to 30 PD. The Titmus stereopsis test was performed and none of the patients recognized it. Fusion was also not detected. The same examiner, who was masked to the diagnosis and to the planned surgical procedure, performed all measurements.

Objective evaluation of eye torsion was performed by retinography one week before and one month after the surgery. Mydriasis was achieved with instillation of one drop of tropicamide $1 \%$ in each eye. The head of the patient was stabilized in the chin and forehead rests and eye height was regulated according to the markings in the retinographer. The examiner took care to observe that the patient's head was straight while being photographed; however, no special head brace or leveling device was used. The patient was asked to look at the internal target of the instrument. With the help of a circle-shaped device on the screen of the instrument, the examiner established the position of the optic disc and of the macula in relation to the posterior pole, placing the fovea at the center of the device. The image obtained was used to measure the torsion angle with a software applied in architecture, Autocad $2009^{\circledR}$ (Autodesk, Inc. San Rafael, California). Four lines were drawn tangent to the upper, medial, lower and lateral extremities of the optic disc, forming a rectangle, and two diagonal lines crossing the internal angles of the figure. This was the manner to determine the center of the disc, through which a horizontal line was drawn parallel to the upper and lower horizontal lines of the rectangle (Figure 1 A). Then, another line was drawn connecting the center of the optic disc to the center of the fovea, and the angle formed by the two lines was measured, which was called the "torsion angle" (Figure 1 B). The normal location of the fovea was considered to be between the center of the optic disc and a $7.25^{\circ}$ angle below the horizontal line that crosses the center, according to the study performed by Bixenman and Von Noorden ${ }^{(2)}$. For the sake of convention, positive angles were established to be those located above the horizontal line that crosses the center of the optic disc, and negative angles were those located below that line. Pre- and postoperative results were compared. The examiner responsible for the performance of the measurements was blinded to the diagnosis or to the performance of a previous surgery.

The non-parametric Wilcoxon test as used for statistical analysis, for the purpose of comparing the values observed before and after the surgery for each patient. The test was applied separately in the right eye and in the left eye. The same test was applied to compare the right and the left eye for absolute variations observed between the values of the angles measured before and after the surgery. The significance level was established as 0.05 or $5 \%$. Because the current study presented non-parametric data, medians were used.

\section{RESULTS}

Patients presented visual acuity with optical correction varying from $20 / 30$ to $20 / 20$ in both eyes, and no changes were observed with regard to biomicroscopy and fundus examination.

There was a statistically significant difference $(p<0.05)$ between the preoperative torsion angles in the right eyes and in the left eyes; thus, statistical analysis was performed separately for each eye. Table 1 shows the results of angle analyses, and figures $2 \mathrm{~A}, \mathrm{~B}, \mathrm{C}$, and D represent an illustrative example of the results.

Despite the decrease in the angles, six of the 10 right eyes remained in intorsion, three remained within the normal range $\left(0^{\circ}\right.$ to $\left.7.25^{\circ}\right)$, and one presented extorsion $\left(-13,16^{\circ}\right)$. In the left eyes, three remained in intorsion, four presented extorsion $\left(-8.29^{\circ},-9.19^{\circ},-15.80^{\circ},-16.86^{\circ}\right)$ and three remained within normal limits.

The median A-pattern before surgery was 22.5 PD, and zero after surgery, indicating a mean correction of 22.5 PD $(p=0.01)$. The change in superior oblique muscle overaction was statistically significant in both right and left eyes $(p=0.01)$; however, there was no difference with regard to inferior oblique underaction $(p=0.06 / p=0.12)$.

There was no correlation between the values of A-pattern, superior oblique overaction, inferior oblique underaction and the degree of preoperative torsion.

\section{DISCUSSION}

Objective evaluation of eye torsion may be performed with funduscopy and eye fundus photography; the latter has been shown to be effective for quantitative analysis of the angle between the fovea and the optic disc center in a study performed by Morton et al. ${ }^{(5)}$.

In our study, patients were photographed in a primary gaze position, in monocular vision, with a fixating gaze being photographed. According to Kushner et al. ${ }^{(3)}$ there was no significant clinical diffe-
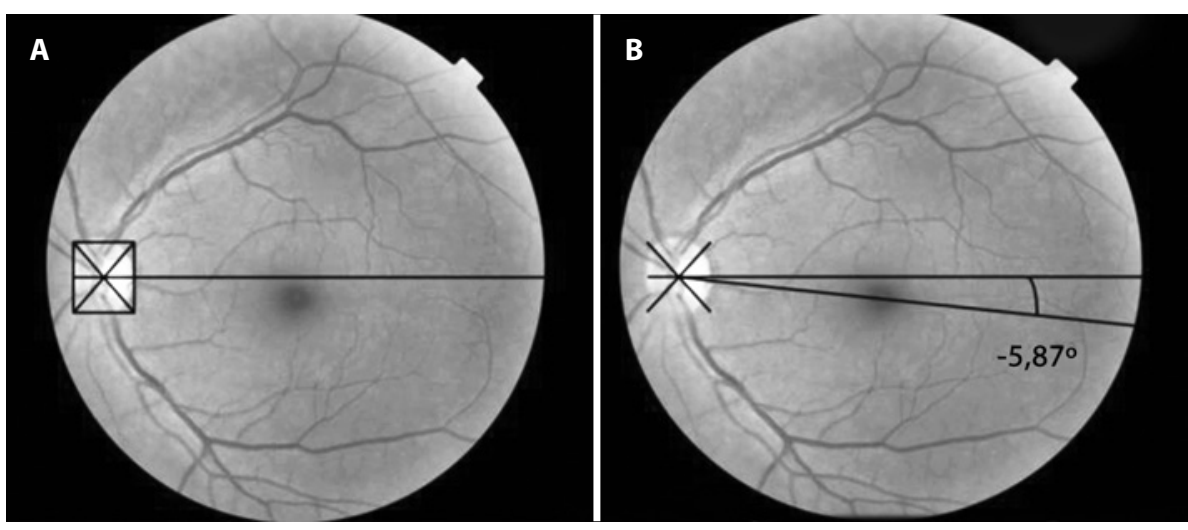

Figure 1. A) Determination of the optic disc center: horizontal line crossing the intersection of the diagonal lines to the center of the optic disc. B) Torsion angle between the centers of the fovea and of the optic disc. 
Table 1. Patients submitted to bilateral tenectomy of the superior oblique muscle (SO) according to the A-pattern, SO overaction, inferior oblique underaction values, to the torsion angles measured in degrees in the pre and postoperative periods, and according to the absolute variation values of these angles

\begin{tabular}{|c|c|c|c|c|c|c|c|c|c|}
\hline \multirow{2}{*}{$\begin{array}{l}\text { Right eyes } \\
\text { patients }\end{array}$} & \multicolumn{4}{|c|}{ Pre-operative } & \multicolumn{4}{|c|}{ Post-operative } & \multirow[b]{2}{*}{ Torsion angle variation } \\
\hline & $A(\Delta)$ & SO & 10 & Torsion angle & $A(\Delta)$ & OS & 10 & Torsion angle & \\
\hline 1 & 25 & +1 & 0 & 4.31 & -15 & -1.5 & +2 & -13.16 & 17.47 \\
\hline 2 & 20 & +2 & 0 & 4.87 & 0 & 0 & 0 & -1.57 & 6.44 \\
\hline 3 & 25 & +3 & 0 & 7.57 & 0 & 0 & 0 & 4.22 & 3.35 \\
\hline 4 & 20 & +2 & -1.5 & 11.53 & 13 & +2 & -1 & 6.78 & 4.75 \\
\hline 5 & 30 & +1 & -2 & 11.83 & 0 & 0 & 0 & 2.64 & 9.19 \\
\hline 6 & 25 & +3 & -2.5 & 19.36 & 0 & 0 & 0 & 5.97 & 13.39 \\
\hline 7 & 30 & +3 & -3 & 1.36 & -5 & +3 & -1.5 & -3.61 & 4.97 \\
\hline 8 & 20 & +2 & -2 & 4.05 & 5 & +1 & -1 & 1.04 & 3.01 \\
\hline 9 & 15 & +1.5 & 0 & -2.82 & -12 & +1 & 0 & -6.59 & 3.77 \\
\hline 10 & 15 & +1.5 & 0 & 6.26 & 0 & +1 & 0 & 3.75 & 2.61 \\
\hline Median & 22.5 & +2 & -0.75 & 5.56 & 0 & 0.5 & & 1.84 & 4.86 \\
\hline P & \multicolumn{9}{|c|}{0.012} \\
\hline Left eyes & \multicolumn{4}{|c|}{ Pre-operative } & \multicolumn{4}{|c|}{ Post-operative } & \\
\hline patients & $A(\Delta)$ & So & 10 & Torsion angle & $A(\Delta)$ & OS & 10 & Torsion angle & Torsion angle variation \\
\hline 1 & 25 & +2 & 0 & 7.21 & -15 & +2 & 0 & -15.80 & 23.01 \\
\hline 2 & 20 & +2 & 0 & 1.43 & 0 & 0 & 0 & -8.29 & 9.72 \\
\hline 3 & 25 & +3 & 0 & 0.66 & 0 & 0 & 0 & -1.50 & 2.16 \\
\hline 4 & 20 & +2 & 0 & 8.43 & 13 & +1 & 0 & 2.65 & 5.78 \\
\hline 5 & 30 & +2 & -2 & 10.61 & 0 & 0 & 0 & -1.48 & 12.09 \\
\hline 6 & 25 & +3 & -2.5 & 2.53 & 0 & 0 & 0 & -4.73 & 7.26 \\
\hline 7 & 30 & +3 & -3 & -3.43 & -5 & +1 & 0 & -9.19 & 5.76 \\
\hline 8 & 20 & +2 & -2 & -1.51 & 5 & +1 & -1 & -16.86 & 15.30 \\
\hline 9 & 15 & +2 & 0 & 5.91 & -12 & 0 & 0 & 1.29 & 4.62 \\
\hline 10 & 15 & +1.5 & 0 & 4.33 & 0 & +2 & 0 & 2.23 & 2.10 \\
\hline Median & 22.5 & +2 & 0 & 3.43 & & & 0 & -3.12 & 6.52 \\
\hline P & \multicolumn{9}{|c|}{0.01} \\
\hline
\end{tabular}

$\mathrm{A}=\mathrm{A}$-pattern in prism dioptries; OS= superior oblique muscle action (+= over; -= underaction); $1 \mathrm{O}=$ inferior oblique muscle $(+=$ over; $=$ underaction); Preop $=$ preoperative; Postop $=$ postoperative; $(-)=$ angles above the horizontal line that crosses the optical disk center; values without a signal mean that the angles are below that line. Wilcoxon Test; $p=s t a t i s t i c a l ~ s i g n i f i c a n c e ~(<0,05)$.
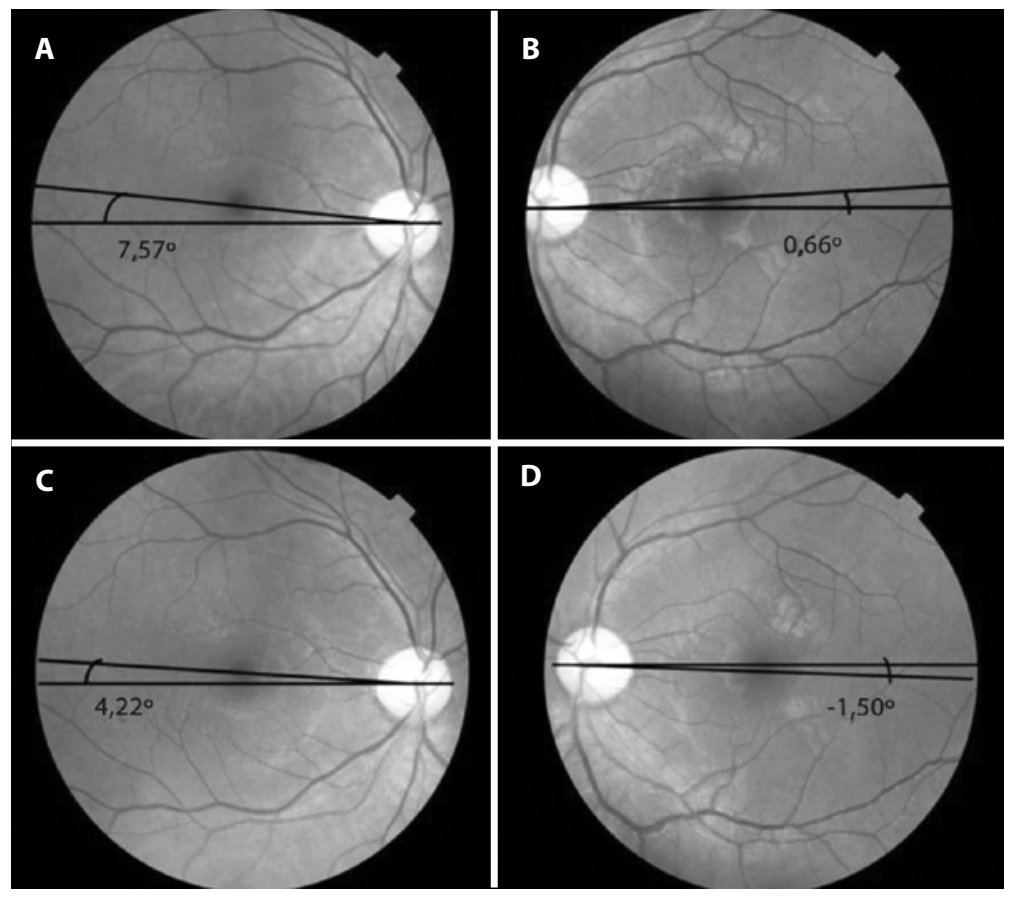

Figure 2. A) Right eye: preoperative torsion angle. B) Left eye: preoperative torsion angle. C) Right eye: postoperative torsion angle. D) Left eye: postoperative torsion angle. 
rence when the photograph was taken with the contralateral eye looking at the external target. Consequently, objective torsion may be studied with either of the eyes looking fixedly at a target.

The Maddox rod test was not performed in the present study because the subjective test results may show absence of torsion due to sensorial adaptations, even if objective tests show changes in eye torsion ${ }^{(9,12,16-18)}$. This is because spatial adaptation offsets the torsion of the image, particularly in patients with congenital torsion alterations or changes acquired very early in life ${ }^{(18)}$.

Among superior oblique weakening techniques, those performed medially to the superior rectus provide greater effect compared with the lateral approach technique, and may cause complications such as superior oblique palsy ${ }^{(6)}$. Scleral disinsertion and tenectomy performed laterally to the superior rectus are the preferred techniques for small and medium overactions of the superior oblique ${ }^{(9)}$. In our study, patients had bilateral superior oblique overaction, varying from $2+$ to $3+$, and the technique used was tenectomy, performed laterally to the superior rectus ${ }^{(11)}$ in both eyes, which acts on both anterior and posterior fibers, thus reducing intorsion. This procedure did not involve risk of permanent diplopia, although some patients ended up with significant excyclotorsion and inferior oblique overaction, since no patients exhibited stereopsis or fusion.

Before surgery, the ocular torsion angles varied from $0.66^{\circ}$ to $19.36^{\circ}$ above the horizontal line that crosses the geometric center of the optic disc, which is a sign of intorsion according to the study performed by Bixenman and Von Noorden ${ }^{(2)}$, who found that the normal location of the fovea is between the center of the optic disc and a $7.25^{\circ}$ angle below the center. As we considered the angles below the horizontal line that crosses the center of the optic disc to be negative, normal values for the position of the fovea are between zero and $-7.25^{\circ}$; extorsion is present when the angle is greater than that value. Bixenman and Von Noorden ${ }^{(2)}$ also established the normal position of the fovea, located at one-third of the disk diameter below the horizontal line that crosses the optic disc center, creating a mean angle of $7.25^{\circ}$ with that line. Guyton ${ }^{(12)}$ described a grading method that classifies the torsion with respect to the optic disc divided into eight parts. In this case, the normal position of the fovea is located $9^{\circ}$ below the optic disc center, which corresponds to the union between the central and the lower thirds.

Our results show that full superior oblique tenectomy acted as an extorsional surgery on the torsion fibers of this muscle, as all of the eyes exhibited a statistically significant difference between the angles before and after surgery. Even eyes that remained in intorsion after surgery were considerably extorted after the tenectomy; for example, the right eye of patient 6 presented a torsion angle variation of $13.39^{\circ}$; however, considering that the final angle was $5.97^{\circ}$, it still remained in intorsion. The mean correction of intorsion was $4.86^{\circ}$ and $6.52^{\circ}$ in the right and left eyes, respectively; these results are similar to those obtained by Roizen et al. ${ }^{(15)}$, who found that anterior fibers tenectomy corrected $5.2^{\circ}$ of intorsion. Sharma et al. ${ }^{(14)}$, in turn, showed that the posterior tenectomy technique did not change eye torsion, with non-statistically significant postoperative results ( $p=0.81$ in the right eye and $p=0.09$ in the left eye), and concluded, therefore, that selective weakening of posterior fibers of the superior oblique muscle only affects the functions of downgaze and abduction of the eye.
In our study, there was considerable variation in surgical torsion changes. This could be correlated with the severity of pre-operative torsion; however, if patient 1 (right eye: pre-operative torsion angle +4.31, post-operative torsion angle-13.16; variation: 17.47) is compared with patient 6 (right eye: pre-operative torsion angle +19.36 , post-operative torsion angle +5.97; variation: 13.39), the former hypothesis becomes invalid. This variation may be due to the lack of knowledge of the position of reinsertion of the tendon after tenectomy. According to Souza-Dias and Prieto-Diaz ${ }^{(7)}$, the closer to the trochlea the superior oblique weakening is performed, the greater the effect. If the tendon is sectioned adjacent to the medial margin of the superior rectus, the weakening effect is smaller. In addition, there are other, unknown factors in the creation of A-patterns that may be related to this variation.

The present study was limited by a small sample size. In addition, it would be interesting to consider a longer follow-up period in future studies.

In conclusion, superior oblique tenectomy caused a mean extorsion of $4.86^{\circ}(p=0.12)$ in the right eyes and $6.52^{\circ}(p=0.1)$ in the left eyes, thus reducing the incycloduction observed with overaction of this muscle.

\section{REFERENCES}

1. Felius J, Locke KG, Hussein MA, Jr DRS, Stager DR. Photographic assessment of changes in torsional strabismus. JAAPOS. 2009;13(6):593-5.

2. Bixenman WW, Von Noorden GK. Apparent foveal displacement in ciclotropia. Ophthalmology. 1982;89(1):58-63.

3. Kushner BJ, Hariharan L. Observations about objective and subjective ocular torsion. Ophthalmology. 2009;116(10):2001-9.

4. Kushner BJ: Effect of ocular torsion on $A$ and $V$ patterns and apparent oblique muscle overaction. Arch Ophthalmol. 2010;128(6):712-18.

5. Morton GV, Lucchese N, Kushner BJ. The role of fundoscopy and fundus photography in strabismus diagnosis. Ophthalmology. 1983;90(10)1189-92.

6. Prieto-Diaz J, Souza-Dias C. Estrabismo. $4^{\text {th }}$ ed. São Paulo: Santos; 2002.

7. Souza-Dias C. Coleção de manuais básicos CBO/Estrabismo. São Paulo: Cultura Médica;1999.

8. Berke RN. Tenotomy of the superior oblique muscles for hypertropia. Arch Ophthalmol. 1947;38(5):605-44.

9. Scott WE, Jampolsky J, Redmond MR. Superior oblique tenotomy: indications and complications. Int Ophthalmol Clin. 1976;16(3):151-9.

10. Prieto-Diaz J. Posterior tenectomy of the superior oblique. J Pediatr Ophthalmol Strabismus. 1979;16(5):321-3.

11. Souza-Dias C. Full tenectomy of the superior oblique muscle close to the scleral insertion for the correction of "A" anisotropia. Proceedings of the Fifth Meeting. ISA-V-Rome. 1986. p.429-36.

12. Guyton DL. Clinical assessment of ocular torsion. Am Orthoptic J. 1983;33:7-15.

13. Santiago AP, Isenberg SJ, Apt L, Roh YB. The effect of anterior transposition of the inferior oblique muscle on ocular torsion. JAAPOS. 1997;1(4):191-6.

14. Sharma P, Saxena R, Rao BV, Menon V. Effect of posterior tenectomy of the superior oblique on objective and subjective torsion in cases of superior oblique overaction. J Pediatr Ophthalmol Strabismus. 2005;42(5):284-9.

15. Roizen A, Velez FG, Rosenbaum AL: Superior oblique anterior tenectomy. JAAPOS. 2008;12(1):54-7.

16. Guyton DL. Ocular torsion reveals the mechanisms of cyclovertical strabismus - the Weisenfeld Lecture. Invest Ophthalmol Vis Sci. 2008;49(3):847-57.

17. Gobin MH. Desinsertion of the superior oblique in "A" pattern strabismus. Doc Ophthalmol. 1997:44(1):193-202.

18. Von Noorden GK. Binocular vision and ocular motility. St Louis: Mosby; 2002. 